\title{
Predictors of treatment adherence in
} patients with chronic disease using the Multidimensional Adherence Model: unique considerations for patients with haemophilia

Karen Strike, Anthony Chan, Alfonso lorio, Monica R. Maly, Paul W. Stratford, Patricia Solomon

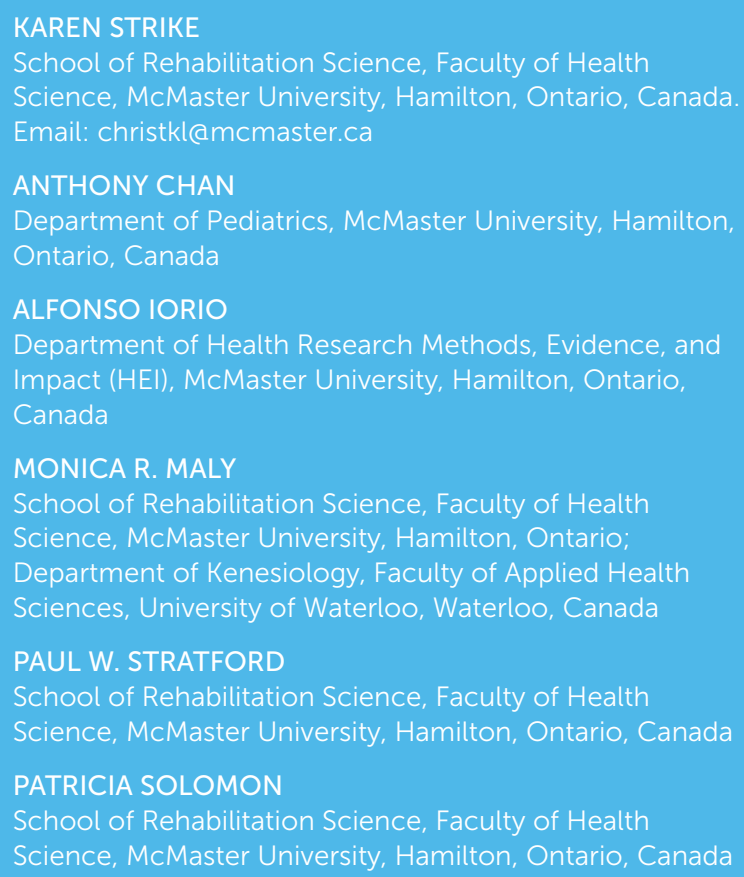

Introduction: Adherence to treatment recommendations in patients with chronic disease is complex and is influenced by numerous factors. Haemophilia is a chronic disease with reported levels of adherence ranging from 17-82\%. Aim: Based on the theoretical foundation of the World Health Organization Multidimensional Adherence Model,

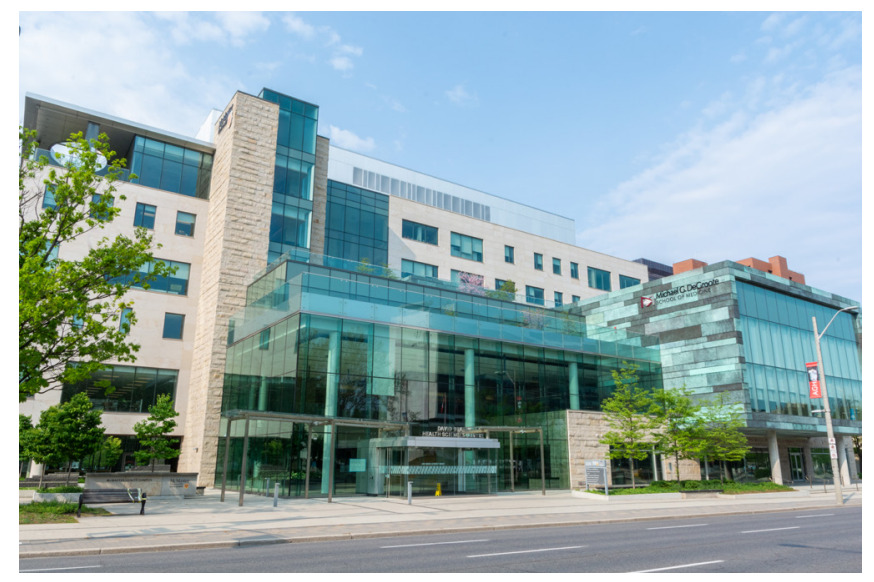

Adherence to prophylaxis among people with haemophilia is known to be variable. A retrospective study of patients treated at an urban haemophilia treatment centre in Canada suggests there may be predictors of adherence beyond the WHO's inclusive Multidimensional Adherence Model.

the objective of this study was to identify the best combination of the variables infusion frequency, annualised bleed rate, age, distance to haemophilia treatment centre (HTC) and Haemophilia Joint Health Score (HJHS), to predict adherence to treatment recommendations in patients with haemophilia $A$ and $B$ on home infusion prophylaxis in Canada. Methods: A one-year retrospective cohort study investigated adherence to treatment recommendations using two measures: 1) subjective report via home infusion 
diaries, and 2) objective report of inventory ordered from Canadian Blood Services. Stepwise regression was performed for both measures. Results: Eighty-seven patients with haemophilia $A$ and $B$, median age 21 years, were included. Adherence for both measures was $81 \%$ and $93 \%$ respectively. The sample consisted largely of patients performing an infusion frequency of every other day (34\%). Median scores on the HJHS was 10.5; annualised bleed rate was two. Distance to the HTC was $51 \mathrm{~km}$. Analysis of the objective measure weakly supported greater infusion frequency as a treatmentrelated factor for the prediction of lower adherence, however the strength of this relationship was not clinically relevant $(R 2=0.048)$. For the subjective measure, none of the explanatory variables were significant. Conclusion: Adherence is a multifaceted construct. Despite the use of theory, most of the variance in adherence to treatment recommendations in this sample of patients with haemophilia remains unknown. Further research on other potential predictors of adherence, and possible variables and relationships within factors of the MAM is required.

Keywords: Treatment adherence and compliance; chronic disease; haemophilia A; haemophilia B; model, theoretical; prophylaxis

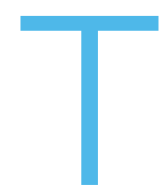

he World Health Organization (WHO) defines adherence as "the extent to which a person's behaviour - taking medication, following a diet and/or executing lifestyle changes, corresponds with agreed recommendations from a health care provider" ${ }^{\prime \prime 11}$. The WHO suggests that improving adherence to treatment recommendations for patients with chronic disease may have a greater impact on patient outcomes than any improvement or innovation in medical treatment ${ }^{[1]}$. Approximately $60 \%$ of patients with chronic disease have less than optimal levels of adherence ${ }^{[2]}$. In 2018, the annual costs of medication non-adherence was estimated to be approximately $\$ 290$ billion in the USA, $€ 25$ billion in Europe and $\$ 7$ billion in Australia ${ }^{[3]}$. Given fiscal restraints within health care systems, it is prudent to understand the factors that contribute to low levels of adherence in patients with chronic disease. Coupling this information with a theoretical foundation can then allow for research into interventions to improve adherence ${ }^{[4]}$.

Haemophilia is a chronic disease in which blood cannot clot due to the absence or deficiency of a clotting factor protein. The two most common types are haemophilia A (Factor VIII deficiency) and haemophilia B (Factor IX deficiency) ${ }^{[5]}$. Haemophilia has an estimated frequency of one per 10,000 births and there are approximately 400,000 people with haemophilia worldwide ${ }^{[5]}$. For both haemophilia A and B, joint bleeding accounts for $70 \%$ to $80 \%$ of all bleeding episodes ${ }^{[5]}$. Although any joint may be affected, hinge joints, particularly the ankles, knees and elbows, are the most commonly involved ${ }^{[5]}$. Treatment for haemophilia involves the intravenous replacement of the missing clotting factor using various treatment regimens. Prophylaxis, also known as regular replacement therapy, is treatment given continuously for 52 weeks per year to prevent or reduce the risk of bleeding ${ }^{[6,7]}$. On-demand treatment is when factor is given in response to a bleeding episode. In comparison to on-demand treatment, prophylaxis offers greater protection from life-threatening haemorrhages, prevention of joint bleeding, lower annualised bleed rates, and improved quality of life ${ }^{[]]}$. Dosage and frequency of prophylaxis infusions is determined by a number of factors including, bleeding phenotype, venous access, goals of treatment, age of the patient, and the costs of treatment ${ }^{[7]}$. With the recent advances in extended half-life factor products and the ability to provide individualised prophylaxis recommendations through population pharmacokinetic modelling, prophylaxis recommendations are also being guided by minimum trough levels. Currently, the goal for many developed countries is a minimum trough level of $1-5 \%$ to prevent breakthrough bleeding ${ }^{[7]}$. Given the required frequency of infusions to achieve the goals of prophylaxis, and to ensure prompt access to treatment in the event of a bleed, patients/caregivers are trained to perform factor infusions at home.

While home infusion offers many benefits, adherence to prophylaxis recommendations is variable ${ }^{[8]}$, potentially leading to recurrent joint and muscle bleeding ${ }^{[9]}$. Joint disease affects $90 \%$ of people with severe haemophilia and contributes the greatest cost and morbidity in the haemophilia population ${ }^{[10]}$. Prophylaxis improves joint outcomes by decreasing the number of bleeding episodes and preventing the development of chronic arthropathy ${ }^{[11]}$. Effectiveness of prophylaxis is influenced by dosage and frequency but more importantly by patients adhering to factor treatment recommendations ${ }^{[11]}$. Adherence to treatment recommendations is a complex phenomenon influenced by personal and societal variables. In patients with haemophilia, reported levels of adherence ranges from $17-82 \%{ }^{[12-14]}$. Reasons for the large variation in levels of adherence include both measurement and treatment-related issues. 
Measurement-related issues include a lack of accepted definition of adherence and the different measures used to calculate adherence, while treatment-related issues may include the time-consuming nature of prophylaxis treatment, the cost of factor therapy, the perceived lack of benefit, frequent venous access, lifestyle disruptions, cooperation of the patient, and the complexity and duration of treatment ${ }^{[12-14]}$. Given the number of variables and wide range of adherence, further research is needed to understand the relationship between adherence and clinical outcomes ${ }^{[12]}$. Crucial to this process is the systematic evaluation of the predictors of treatment adherence using theoretical models as a foundation ${ }^{[15]}$.

Investigation into the predictors of treatment adherence in chronic diseases, such as haemophilia, should start with a consideration of relevant theory, in order to provide a structure to generate hypotheses, and to conceptualise ideas to organise, predict and explain certain events or behaviours ${ }^{[4]}$. Ecological theory takes into account the influence of different levels of the health care system on patient behaviour ${ }^{[16]}$. The WHO Multidimensional Adherence Model (MAM) is an ecological model that combines intrapersonal, interpersonal, organisational, policy, and community barriers, to understand adherence behaviour ${ }^{[15,16]}$.

The MAM framework classifies barriers to adherence into five dimensions: health care team/health system, socioeconomic factors, patient-related factors, condition-related factors and treatment-related factors (see Figure 1) ${ }^{[15,16]}$. The MAM also highlights the need for a multidisciplinary approach to adherence, which fits well within the comprehensive model of care that has been adopted in the treatment of haemophilia in Canada ${ }^{[17]}$.

This study is the first to investigate treatment adherence in patients with haemophilia using the MAM. The primary purpose was to determine which combination of factors (infusion frequency, annualised bleed rate, age, distance to the haemophilia treatment centre (HTC), Haemophilia Joint Health Score (HJHS)) best predicts adherence to treatment recommendations in patients with haemophilia $A$ and $B$ on home infusion prophylaxis in Canada using two measures of adherence. The secondary purpose was to determine which of the two measures of adherence produced the most explanatory model.

\section{METHODS}

\section{Study design}

This was a one-year retrospective cohort study.

\section{Data sources}

Data were extracted from the medical record and the Canadian Bleeding Disorders Registry (CBDR). The CBDR is a collaboration among the Association of Hemophilia Clinic Directors of Canada (AHCDC), McMaster University and the Australian National Blood Authority, with support from the Canadian Hemophilia Society. The CBDR is a national registry and clinical database for patients with bleeding disorders, and is used by HTCs to support patient care, improve research and monitor factor concentrate utilisation ${ }^{[18]}$. The CBDR is linked to MyCBDR, a web-based home

Figure 1: World Health Organization Multidimensional Adherence Model ${ }^{[15]}$

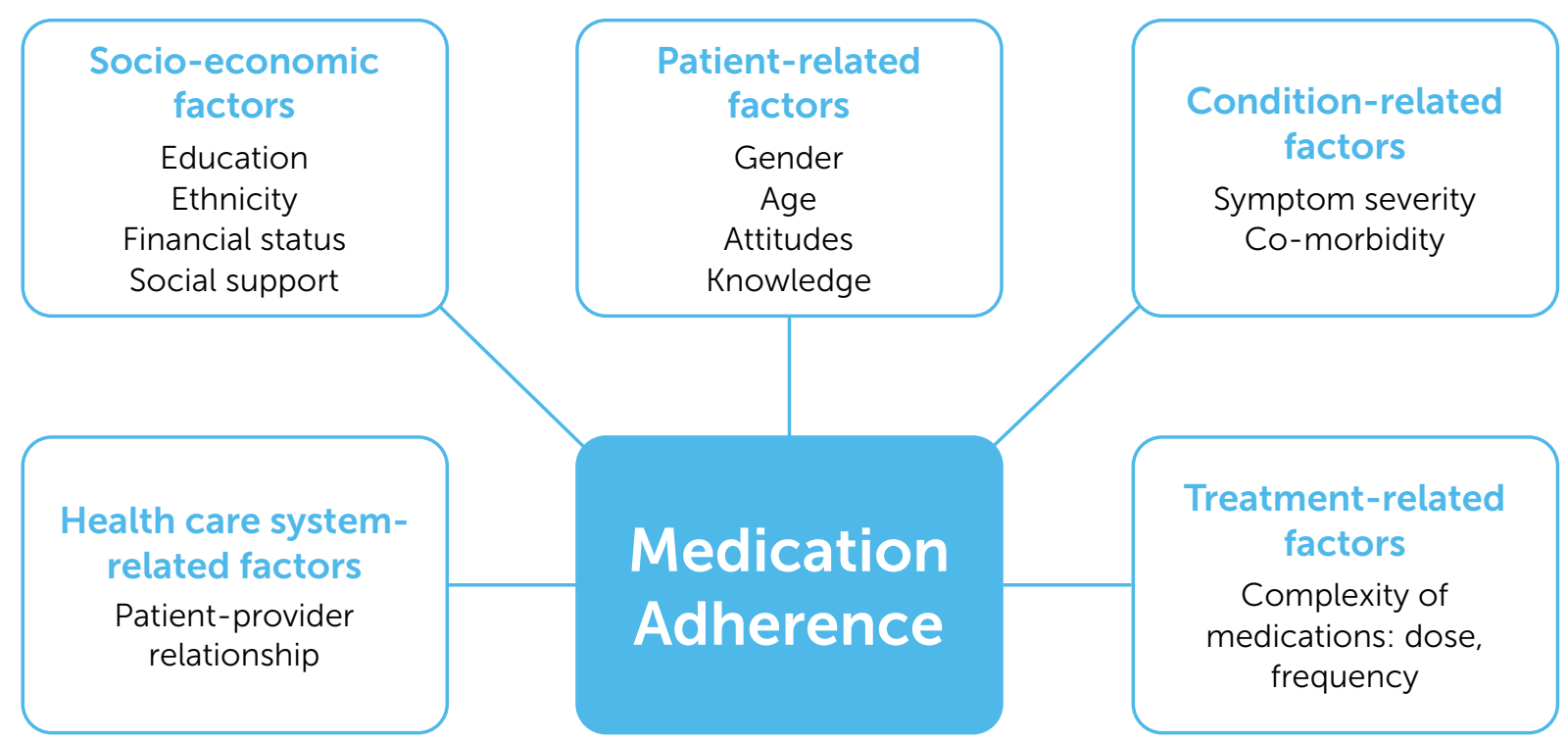


infusion patient diary. The AHCDC owns the CBDR and MyCBDR; McMaster University manages the system on behalf of the AHCDC and regularly performs data cleaning initiatives to ensure fidelity of the data.

\section{Setting}

The HTC Comprehensive Care Model (CCM) is the standard of care for haemophilia in Canada. The CCM focuses on family-centred care and the prevention of the acute and chronic manifestations of a bleeding disorder through education and coordination of services available within the HTC and the surrounding region ${ }^{[19]}$. For this study, data were accessed for patients from a large urban HTC in Canada.

\section{Study procedures}

Retrospective data were extracted and anonymised from the CBDR, MyCBDR and the medical record for the 2017 calendar year. Ethics approval was obtained from the Hamilton Integrated Research Ethics Board.

\section{Participants}

All patients with haemophilia A or B who were prescribed prophylaxis via home infusion were included. Patients receiving prophylaxis in long-term care, via community resources and those in research studies were excluded.

\section{Sample size}

Although sample size formulae exist with respect to evaluating the entire model (e.g., is the R-squared different from a null value) and whether the addition of one or more variables (the number being specified by the investigator a priori) add to its predictive ability given the presence of covariates, there is no accepted formula for sample size for stepwise regression. An important consideration with stepwise regression is the number of 'behind the scenes' multiple comparisons and phantom degrees of freedom applied to come up with the final model ${ }^{[20]}$. This has the potential, based on chance, to include variables that are truly not predictive of outcome, as well as to miss variables that are truly predictive of outcome. Accordingly, the stability of a given model is determined by cross-validation and the calculation of shrinkage. Therefore, a convenience sample of all eligible subjects was used and an acceptable level of shrinkage was set at $10 \%$ or less ${ }^{[21]}$.

\section{Variables}

\section{Dependent variable: treatment adherence}

There is currently no gold standard for the evaluation of medication adherence ${ }^{[1]}$. In clinical research, adherence is often measured by the number of doses used by the patient compared to the number of doses prescribed by the physician ${ }^{[7]}$. In this study, two measures of adherence that are readily available in the CBDR were used. The first, a subjective report, was calculated as a percentage of reported factor usage via home infusion diaries divided by the physician prescribed dose for the 2017 calendar year. The second, an objective report, was calculated as a percentage of factor inventory ordered by the patient via the Canadian Blood Services divided by the physician prescribed dose for the 2017 calendar year. The subjective report assumed that if there was no record, then the infusion was not given. The objective report assumed that all factor ordered for home infusion was for prophylaxis and was used as prescribed. For both measures, the physician prescribed dose was for prophylaxis treatment recommendations. Given these assumptions, both methods were used to determine which method resulted in the most explanatory model.

\section{Explanatory variables}

The candidate variables were chosen to align with the MAM.

Distance from home (health care system-related factors) Health care system-related factors include systems, organisations or access to health care professionals who provide care to patients ${ }^{[17,22]}$. The amount of time spent within the HTC and the relationship with the team is strongly associated with adherence ${ }^{[23]}$. Access to the $\mathrm{HTC}$, as measured by the distance in kilometres $(\mathrm{km})$ from the patient's home address recorded in the CBDR to the address of the HTC, was calculated and investigated as a health care system-related factor. For this study, it was hypothesised that burden of travel to the HTC would limit time spent in the HTC. It has been shown in the literature that for patients with chronic disease, transportation is essential for ongoing health care and access to treatment and medication ${ }^{[24]}$. Burden of travel has been defined by the US Department of Transportation as travelling greater than 30 minutes or 30 miles $/ 48 \mathrm{~km}$ to a health care provider ${ }^{[24]}$.

Age (socioeconomic and patient-related factors) Socioeconomic factors relate to social and economic status ${ }^{[17,22]}$. Patient-related factors can include patient characteristics such as sex, age, attitudes and beliefs ${ }^{[17,22]}$. In the current study, age is used to represent both domains. Age can represent the availability of social supports as paediatric patients have family/caregivers trained to assist with home infusions while adult patients 
perform self-infusion. Adolescence and the transition to adult care is a particularly important timeframe as this period is often associated with increased independence in disease management with the transfer of responsibilities from the caregiver to the patient ${ }^{[25]}$. Adolescence is also a time of personal change, which may impact adherence to prophylaxis treatment ${ }^{[25]}$. During this transition, it has been found that $41 \%$ of patients with haemophilia aged $13-25$ years did not follow the prescribed treatment recommendations ${ }^{[26]}$. In a study of nurse-reported data, Geraghty et al. found levels of adherence of $90 \%$ in patients aged $0-12$ years, $54 \%$ in patients aged $13-18$, and $36 \%$ in patients aged $19-28$ years $^{[27]}$. A Canadian survey suggests that paediatric patients have higher levels of adherence than adolescents or adults ${ }^{[28,29]}$. Increasing age is also associated with lower levels of adherence ${ }^{[23]}$. Age was calculated in years as of January 1, 2017.

\section{Annualised bleed rate and HJHS (condition-related} factors)

Condition-related factors are characteristics such as symptom severity and comorbidity ${ }^{[17,22]}$. Symptoms, such as bleeding episodes and pain from chronic arthropathy, have been shown to be both a positive and negative motivator for adherence in haemophilia [23,30]. The study used the annualised bleed rate (ABR), or number of bleeds over one year, as a measure of disease activity. ABR is a patient-reported measure and potentially limited by patients' failure to accurately report a painful episode, subclinical bleeding and social desirability ${ }^{[31]}$. However, ABR is still a widely used outcome of treatment efficacy in the majority of clinical trials in haemophilia. Typically, patients on prophylaxis will have a mean ABR of $3.27^{\text {[32] }}$.

Joint disease, once established, is progressive and irreversible ${ }^{[33]}$. Poor adherence to treatment recommendations is associated with worse joint outcomes $^{[32]}$ and greater symptom severity. The Haemophilia Joint Health Score (HJHS) is an objective measure of joint health in patients with haemophilia ${ }^{[34-37]}$. Scores range from 0-124 with higher scores indicating worse joint disease ${ }^{[34,35]}$. The HJHS demonstrates acceptable psychometric properties in paediatrics including excellent test-rest $(I C C=0.89)$ inter-observer $(\mathrm{ICC}=0.83)$ reliability, and internal consistency (Cronbach's alpha $=0.86)^{[35]}$. With regard to construct validity, the HJHS shows good ability to discriminate between known groups of patients and it correlates appropriately with bleeding rates ( $r s=0.50$ ) and physician global assessment of joint health $(r s=0.42)^{[34,36]}$. Criterion validity has been demonstrated and the HJHS correlates well with radiographic changes and favourably with the World Federation of Hemophilia Physical Examination Score when discriminating known groups (63-97\% more efficient) ${ }^{[34,36,37]}$. A validation study in adults is currently underway ( $\mathrm{N}$. Zourikian, codeveloper of the HJHS, oral communication, May 2019). For this study, one HJHS score for the 2017 calendar year was extracted from the CBDR. In the event that two scores were completed, the higher score was used.

Infusion frequency (treatment-related factors) Treatment-related factors are associated with treatments or influence a patient's willingness to receive treatment ${ }^{[17,22]}$. The time required for prophylaxis has been considered a significant barrier to treatment adherence ${ }^{[32]}$. Infusion frequency typically ranges from once per week to daily. For this study, infusion frequency was assessed categorically with the following groupings: 1 = one infusion/week; 2 = two infusions/week; 3 = three infusions/week; 4 = infusions every other day; 5 = infusion every three days; 6 = infusion every five days; $7=$ daily infusions.

\section{Analyses}

Statistical analyses were conducted with alpha set at 0.05 using Stata v.14. Descriptive statistics were reported as means, standard deviations, 95\% confidence intervals (Cl) for continuous variables given a normal distribution and as medians, quartiles for skewed distributions. Counts and percentages were applied to categorical data. Normality was assessed with the Shapiro Wilk test/histogram. Correlation graph matrix and correlation coefficients were investigated to assess for collinearity and the relationship of the explanatory variables with both measures of adherence. If collinearity was found, one of the explanatory variables was removed. Homoscedasticity was assessed with the Breusch-Pagan test. For the subjective measure, the assumption of normality could not be met and a stepwise regression analysis that applied robust standard errors was completed.

To achieve normality, the objective measure was raised to a power of two and a stepwise regression was performed with 0.05 level of significance for model entry and 0.10 for model removal. Regression diagnostics were performed, and outliers were considered for removal from the data set based on clinical plausibility and influence. Normality of residuals was assessed by the Shapiro-Wilk test/histogram, homoscedasticity via the Breusch-Pagan test and multicollinearity via variance inflation factors. Validation of the final model was 
completed using resampling validation, bootstrapping with 200 samples and shrinkage analysis.

The final response variable variation (R2) and regression diagnostics could determine which measure of adherence results in the most explanatory model. Combining this information with relevant clinical theory and logic, would allow for the selection of the preferred measure of adherence.

\section{RESULTS}

Eighty-seven patients met the inclusion criteria. The median age was 21 years (1st and 3rd quartiles: 10, 36) and consisted largely of patients prescribed a prophylaxis regimen of every other day (34\%). The median, 1st and 3rd quartiles for the subjective measure of adherence was $81 \%(51,98)$. The mean, standard deviation for the objective measure of adherence was $93.55 \%$ (24.75). Median scores on the HJHS was $10.5, A B R$ was two and distance to the HTC was $51 \mathrm{~km}$. Descriptive statistics are presented in Table 1. As there was a high correlation between $\mathrm{HJHS}$ and age $(r 2=0.86), \mathrm{HJHS}$ was removed from both analyses. The correlation matrix is presented in Table 2.

Table 1. Characteristics of outcome and explanatory variables

\begin{tabular}{|c|c|c|c|}
\hline VARIABLE & $\mathbf{N}$ & MEAN/SD & MIN/MAX (\%) \\
\hline \multirow{2}{*}{$\begin{array}{l}\text { Objective measure of } \\
\text { adherence }^{\dagger}\end{array}$} & 87 & $93.55 / 24.75$ & $0 / 154 \%$ \\
\hline & $\mathbf{N}$ & MEDIAN & 1ST \& 3RD QUARTILES \\
\hline $\begin{array}{l}\text { Subjective measure of } \\
\text { adherence }^{\ddagger}\end{array}$ & 87 & 81 & 51,98 \\
\hline $\mathrm{ABR}^{\mathfrak{S}}$ & 87 & 2 & 0,4 \\
\hline Age $^{\pi}$ & 87 & 21 & 10,36 \\
\hline Distance from $\mathrm{HTC}^{\dagger t}$ & 87 & 51 & 26,71 \\
\hline \multirow[t]{2}{*}{$\mathrm{HJHS}^{\ddagger \ddagger}$} & 76 & 10.5 & $1,23.5$ \\
\hline & CATEGORIES & COUNTS & PERCENTAGE OF SAMPLE \\
\hline Infusion frequency 55 & $\begin{array}{l}1 \\
2 \\
3 \\
4 \\
5 \\
6 \\
7\end{array}$ & $\begin{array}{l}8 \\
20 \\
21 \\
30 \\
2 \\
1 \\
5\end{array}$ & $\begin{array}{l}9 \% \\
23 \% \\
24 \% \\
34 \% \\
2 \% \\
1 \% \\
6 \%\end{array}$ \\
\hline
\end{tabular}

Table 2. Correlation matrix

Correlation co-efficient of the explanatory variables with both measures of adherence

\begin{tabular}{|c|c|c|c|c|c|c|c|}
\hline & SUBJECTIVE & OBJECTIVE & HJHS & AGE & FREQUENCY & ABR & DISTANCE \\
\hline Subjective $^{\ddagger}$ & 1.0000 & & & & & & \\
\hline Objective $^{\dagger}$ & 0.4568 & 1.0000 & & & & & \\
\hline $\mathrm{HJHS}^{\ddagger \ddagger}$ & -0.0228 & 0.0651 & 1.0000 & & & & \\
\hline Age & 0.0128 & 0.0375 & 0.8573 & 1.0000 & & & \\
\hline Frequency ${ }^{\S 5}$ & 0.0402 & -0.1959 & -0.0250 & -0.0351 & 1.0000 & & \\
\hline $\mathrm{ABR}^{\mathfrak{J}}$ & -0.0861 & 0.0310 & 0.3438 & 0.2597 & -0.0236 & 1.0000 & \\
\hline Distance $^{\dagger \dagger}$ & 0.0977 & -0.0307 & 0.0442 & 0.0690 & -0.0346 & 0.2038 & 1.0000 \\
\hline
\end{tabular}

Key to Tables 1 and 2

t Objective measure of adherence: percentage of factor inventory ordered by the patient via the Canadian Blood Services divided by the physician prescribed dose

₹ Subjective measure of adherence: percentage of reported factor usage via home infusion diaries divided by the physician prescribed dose

s ABR: annualised bleed rate $=$ number of bleeds a patient experiences over one year

- Age: in years as of January 1, 2017

tt Distance from HTC: distance in kilometres $(\mathrm{km})$ from the patients' home to the haemophilia treatment centre.

\# HJHS: Haemophilia Joint Health Score version 2.1.

gs Infusion frequency with the following legend: 1 = one infusion per week; 2 = two infusions per week; 3 = three infusions per week; 4 =infusions every other day; 5 = infusion every three days; 6 = infusion every five days; $7=$ daily infusions. 
Table 3. Full model stepwise regression for the objective measure of adherence

\begin{tabular}{|c|c|c|c|c|}
\hline VARIABLE & $\beta$-COEFFICIENT & STD ERROR & P-VALUE & $95 \% \mathrm{Cl}$ \\
\hline Frequency & -672.394 & 324.506 & 0.041 & $-1317.599,-27.189$ \\
\hline _cons ${ }^{\dagger}$ & 11536.88 & 1147.566 & 0.000 & $9255.207,13818.54$ \\
\hline
\end{tabular}

${ }^{\dagger}$ _cons $=$ represents the constant or intercept for the regression line

Stepwise regression for the objective measure of adherence

Initial residual analysis showed skewing of the objective adherence measure which was corrected by squaring the dependent variable. Stepwise analysis revealed that age, distance to the HTC and ABR were not statistically significant, leaving infusion frequency $(p=0.041)$ in the final model with an R2 of 0.048 , adjusted R2 of 0.037 (Table 3). However, the shrinkage was $83 \%$, indicating a lack of stability.

Stepwise regression for the subjective measure of adherence

There was severe skewing of the subjective adherence measure with a preponderance of observations showing extremely high adherence. Several transformations were attempted; however, these were unsuccessful. Accordingly, a stepwise regression with robust standard errors was performed. None of the variables were predictive of subjective adherence $(\mathrm{R} 2=0)$.

\section{DISCUSSION}

Adherence is a multifaceted construct and is influenced by many factors. Use of the MAM to conceptualise and select explanatory variables to predict adherence is a strength of this study. Analysis of the objective measure weakly supported the relationship of infusion frequency as a treatment-related factor for adherence. Less frequent infusion regimens are associated with improved adherence for both patients with chronic disease in general and in patients with osteoporosis ${ }^{[38}$. For patients with haemophilia, with the availability of extended halflife products and the possibility of less frequent infusions, patients may become more adherent ${ }^{[32,38]}$. While higher infusion frequencies were associated with lower levels of adherence in this study, infusion frequency explained only $4.8 \%$ of the variance and is not considered clinically relevant. Closely related to infusion frequency is venous access. As factor replacement therapy must be given intravenously, venous access has been shown to be a potential barrier to adherence ${ }^{[1,23]}$. As this study found, a weak association with adherence and infusion frequency, future studies should include methods of venous access as a potential predictor of adherence.
There is no gold standard for the measurement of adherence in patients with haemophilia ${ }^{[39]}$. Various methods of measuring adherence have been reported in previous studies, including patient surveys, physician/nurses perception of adherence ratings, interviews, analysis of infusion diaries, and self-reported questionnaires such as the VERITAS-Pro ${ }^{[28,38-41]}$. However, none of these measures have been validated for patients with haemophilia in Canada, and the heterogeneity in measurement presents barriers to the analysis of predictors of adherence and are important directions for future research.

This study investigated two measures of adherence using data that is readily available in the medical record, the CBDR and home infusion diaries. However, due to the complexity of the adherence construct and the lack of clinical relevance of either model, we were unable to determine which measure provided the most explanatory model. Combining patients with both haemophilia A and B in the sample may affect the generalisability of the results, as patients with haemophilia A have treatment recommendations with a greater frequency of infusions. Further, adherence to prophylaxis over the lifespan further complicates the relationship ${ }^{[42,43]}$. Predictors may be interdependent and there may be commonalities and differences among groups of patients with different levels of adherence. Future analyses using hierarchical regression with patient clustering by adherence, age or diagnosis could provide further insight.

The results indicate that there are other predictors of adherence in patients with haemophilia. Although the MAM aims to be an inclusive model of adherence, this study demonstrates that system-level factors are potentially less predictive of adherence than assumed within a well-organised socialised healthcare system such as that found in Canada. Due to the availability of system-level data in the medical record and the CBDR, the variables used to investigate adherence focused on clinical and demographic factors with a lack of patientrelated variables. Research on adherence in patients with heart failure using the MAM found that the most important and consistent predictor of poor adherence was patient perception of the barriers to adherence, 
such as forgetting medication and the belief that missing a dose will not be harmful [15]. Condition-related factors (i.e. patient function), and socioeconomic factors (i.e. social supports, ethnicity, financial status) were related to at least one indicator of adherence ${ }^{[15]}$. Currently, there is a lack of data on patient perceived barriers to adherence and socioeconomic factors for patients with haemophilia and their families available in the CBDR, and given the lack of variance explained by system level factors, this is a limitation of the current study. A prospective study with the inclusion of the patient's perspective, patient function and socioeconomic factors is an important consideration for future research.

Although, patients in this study demonstrated high levels of adherence, there was a wide range with a minimum/maximum of $0-154 \%$ reported. The high level of adherence in this sample may be due to clinic policy to reduce the amount of factor per order until home infusion records are received. Further, staff demonstrate how home infusion records assist in clinical decision making by engaging patients in the analysis of bleeding episodes. Patient engagement and individualised treatment recommendations improve adherence ${ }^{\text {[32] }}$. Within the HTC, individualisation of care has expanded to include pharmacokinetics with the Web Accessible Population Pharmacokinetic Service ${ }^{[44]}$. Non-adherence in this study was characterised by missed infusions or a lack of ordering sufficient amounts of factor to fulfil prophylaxis recommendations. Bleeding events, overadministration of factor resulting in an adherence rate of greater than $100 \%$, and timing of infusions were not investigated, all of which may have decreased the ability to detect differences in adherence. Future studies that use the amount of factor dispensed as an estimate of adherence should consider using methods that adjust for bleed reporting ${ }^{\text {[31] }}$.

Despite previous research demonstrating adolescence $^{[29]}$, increasing age and lack of experience of symptoms as significant barriers to adherence ${ }^{[23]}$, no relationship was found in this study. Seventy-five per cent of the sample was under 36 years of age and the median age was 21 years. The disproportionate number of zero scores on the $\mathrm{HJHS}(22 \%$, age range 1-13 years) and the median score of 10.5 , makes interpreting the HJHS difficult. The significant collinearity with age and HJHS further influenced the analysis, as it is well known that joint health deteriorates with age. Consistent with the expectation of three bleeds per year in patients prescribed prophylaxis ${ }^{[32]}$, this sample of patients demonstrated a median ABR of two. Therefore, this sample included patients who were younger with minimal joint arthropathy and limited experience of symptoms, which may impact generalisability as the sample may not represent the broader haemophilia population in Canada. Although all eligible patients and data were included in this study, the convenience sample may not be adequately powered and is a possible limitation. Future research with a larger, more heterogeneous population is warranted.

\section{CONCLUSION}

Adherence to prophylaxis is the most effective method of preventing or delaying the development of arthropathy and functional decline in patients with haemophilia ${ }^{[32]}$. This is the first study to investigate the predictors of treatment adherence in patients with haemophilia A or B on prophylaxis using the MAM. In spite of a theoretical approach, a strong methodological and conceptual rationale for the selection of explanatory variables, and the use of two measures of adherence, there were no clinically relevant findings and most of the variance in adherence in this sample remains unknown. Infusion frequency is a possible predictor of adherence that requires further study. It appears that there are other potential predictors of adherence and further research on the possible variables and relationships within factors of the MAM is required.

\section{ACKNOWLEDGEMENTS}

\section{Author contributions}

KS, AC, and PS conceived and designed the study. AC, PS, AI, MRM, and PWS provided mentorship, methodological and statistical support. KS completed the analysis and drafted the manuscript. All authors provided edits and contributed to the final version.

\section{Disclosures}

The authors have advised no interests that might be perceived as posing a conflict or bias.

This paper reports a retrospective study in which no human participants or animals are directly involved.

\section{ORCID}

Karen Strike (iD https://orcid.org/0000-0002-5598-2051 Anthony Chan (D) https://orcid.org/0000-0003-1551-3995 Alfonso lorio (iD https://orcid.org/0000-0002-3331-8766 Monica R. Maly (iD) https://orcid.org/0000-0001-5414-3777 Paul W. Stratford (iD https://orcid.org/0000-0003-4971-2386 Patricia Solomon (iD https://orcid.org/0000-0002-5014-0795 


\section{REFERENCES}

1. World Health Organization. Adherence to long-term therapies: evidence for action. 2003. Available from: https://www.who. int/chp/knowledge/publications/adherence_full_report. pdf?ua=1 (accessed 15 June 2020).

2. Dunbar-Jacob J, Mortimer-Stephens MK. Treatment adherence in chronic disease. J Clin Epidemiol 2001; 54 Suppl 1: S57-60. doi: 10.1016/s0895-4356(01)00457-7.

3. Cutler RL, Fernandez-Llimos F, Frommer M, Benrimoj C, Garcia-Cardenas V. Economic impact of medication nonadherence by disease groups: a systematic review. BMJ Open 2018; 8(1): e016982. doi: 10.1136/ bmjopen-2017-016982.

4. Sirur R, Richardson J, Wishart L, Hanna S. The role of theory in increasing adherence to prescribed practice. Physiother Can 2009; 61(2): 68-77. doi: 10.3138/physio.61.2.68.

5. Srivastava A, Brewer AK, Mauser-Bunschoten EP, et al.; Treatment Guidelines Working Group on behalf of the World Federation of Hemophilia. Guidelines for the management of hemophilia. Haemophilia 2013; 19(1): e1-47. doi: 10.1111/j.1365-2516.2012.02909.x.

6. Blanchette, V., Key NS, Ljung LR, Manco-Johnson MJ, Van Den berg HM, Srivastava A. Definitions in hemophilia: communication from the SSC of the ISTH. J Thromb Haemost 2014; 12: 1935-39. doi: 10.1111/jth.12672.

7. Castaman G,Linari S. Prophylactic versus on-demand treatments for hemophilia: advantages and drawbacks. Expert Rev Hematol 2018; 11(7): 567-76. doi: 10.1080/17474086.2018.1486704.

8. Thornburg CD, Pipe SW. Adherence to prophylactic infusions of factor VIII or factor IX for haemophilia. Haemophilia 2006; 12(2): 198-9. doi: 10.1111/j.1365-2516.2006.01172.x

9. De la Corte-Rodriguez H, Rodriquez-Merchan EC. The role of physical medicine and rehabilitation in haemophilia patients. Blood Coagul Fibrinolysis 2013; 24(1): 1-9. doi: 10.1097/ MBC.0b013e32835a72f3.

10. Pettersson $\mathrm{H}$, Ahlberg A, Nilsson IM. A radiologic classification of hemophilic arthropathy. Clin Orthop Relat Res 1980; 149: 153-9.

11. Thornburg CD, Carpenter S, Zappa S, Munn J, Leissinger C. Current prescription of prophylactic factor infusions and perceived adherence for children and adolescents with haemophilia: a survey of haemophilia healthcare professionals in the United States. Haemophilia 2012; 18(4): 568-74. doi 10.1111/j.1365-2516.2012.02756.x.

12. van Os SB, Troop NA, Sullivan KR, Hart DP. Adherence to prophylaxis in adolescents and young adults with severe haemophilia: a quantitative study with patients. PLoS One 2017; 12(1): e0169880. doi: 10.1371/journal.pone.0169880

13. du Treil S, Rice J, Leissinger CA. Quantifying adherence to treatment and its relationship to quality of life in a wellcharacterized haemophilia population. Haemophilia 2007; 13(5): 493-501. doi: 10.1111/j.1365-2516.2007.01526.x.

14. Hacker MR, Geraghty S, Manco-Johnson M. Barriers to compliance with prophylaxis therapy in haemophilia. Haemophilia 2001; 7(4): 392-96. doi: 10.1046/j.13652516.2001.00534.x.

15. Wu J-R, Moser DK, Chung ML, Lennie TA. Predictors of medication adherence using a multidimensional adherence model in patients with heart failure. J Cardiac Fail 2008; 14(7): 603-14. doi: 10.1016/j.cardfail.2008.02.011.

16. Alghurair SA, Hughes CA, Simpson SH, Guirguis LM. A systematic review of patient self-reported barriers of adherence to antihypertensive medications using the World Health Organization Multidimensional Adherence Model. J Clin Hypertens (Greenwich) 2012; 14(12): 877-86. doi: 10.1111/j.1751-7176.2012.00699.x.

17. De Geest S, Sabaté E. Adherence to long-term therapies: evidence for action. Eur J Cardiovasc Nurs 2003; 2(4): 323. doi: 10.1016/S1474-5151(03)00091-4.

18. Page D, Crymble S, Lawday K, et al. Penny wise, pound foolish: an assessment of Canadian hemophilia/inherited bleeding disorder comprehensive care program services and resources. Haemophilia 2016; 22: 531-36. doi: 10.1111/hae.12913.

19. Barry DG, Hibner S. Care of the chronically ill patient with a bleeding disorder. Rehabil Nurs 2015; 40: 197-206. doi: 10.1002/rnj.129.

20. Babyak MA. What you see may not be what you get: a brief, nontechnical introduction to overfitting in regression-type models. Psychosom Med 2004; 66: 411-21. doi: 10.1097/01. psy.0000127692.23278.a9.

21. Kleinbaum DG, Kupper LL, Nizam A, Muller KE. Applied Regression Analysis and Other Multivariable Methods. 4th ed. 2008, Boston, MA: Duxbury.

22. Leventhal MJE, Riegel B, Carlson B, De Geest S. Negotiating compliance in heart failure: remaining issues and questions. Eur J Cardiovasc Nurs 2005; 4(4): 298-307. doi: 10.1016/j. ejcnurse.2005.04.001.

23. Schrijvers LH, Uitslager N, Schuurmans MJ, Fischer K. Barriers and motivators of adherence to prophylactic treatment in haemophilia: a systematic review. Haemophilia 2013; 19(3): 355-61. doi: 10.1111/hae.12079.

24. Syed ST, Gerber BS, Sharp LK. Traveling towards disease: transportation barriers to health care access. J Community Health 2013; 38(5): 976-93. doi: 10.1007/s10900-013-9681-1.

25. Resseguier N, Rosso-Delsemme N, Beltran Anzola A, et al. Determinants of adherence and consequences of the transition from adolescence to adulthood among young people with severe haemophilia (TRANSHEMO): study protocol for a multicentric French national observational cross-sectional study. BMJ Open 2018; 8(7): e022409. 10.1136/bmjopen-2018-022409.

26. Lindvall K, Colstrup L, Wollter I-M, et al. Compliance with treatment and understanding of own disease in patients with severe and moderate haemophilia. Haemophilia 2006; 12(1): 47-51. doi: 10.1111/j.1365-2516.2006.01192.x.

27. Geraghty S, Dunkley T, Harrington C, Lindvall K, Maahs J, Sek J. Practice patterns in haemophilia A therapy - global progress towards optimal care. Haemophilia 2006; 12(1): 75-81. doi: 10.1111/j.1365-2516.2006.01189.x.

28. Chan AKC, Decker K, Warner M. Adherence with haemophilia treatments: a survey of haemophilia healthcare professionals in Canada. Haemophilia 2011; 17(5): 816-7. doi: 10.1111/j.13652516.2011.02502.x.

29. Bérubé S, Cloutier-Bergeron A, Amesse C, Sultan S. Understanding adherence to treatment and physical activity in children with hemophilia: the role of psychosocial factors. Pediatr Hematol Oncol 34(1); 1-9. doi: 10.1080/08880018.2016.1260669. 
30. De Moerloose P, Urbancik W, Van Den Berg HM, Richards M. A survey of adherence to haemophilia therapy in six European countries: results and recommendations. Haemophilia 2008; 14(5): p. 931-8. doi: 10.1111/j.1365-2516.2008.01843.x.

31. Guedes VG, Corrente JE, Farrugia A, et al. Comparing objective and self-reported measures of adherence in haemophilia. Haemophilia 2019; 25(5): 821-30. doi: 10.1111/hae.13811.

32. Berntorp E. Joint outcomes in patients with haemophilia: the importance of adherence to preventive regimens. Haemophilia 2009; 15(6): 1219-27. doi: 10.1111/j.13652516.2009.02077.x.

33. Trakymien SS. Musculoskeletal pathologies in children with haemophilia evaluated using a standardized physical joint scoring system to assess disability. Eur Oncol Haematol 2011; 7(1): 76-80. doi: 10.17925/EOH.2011.07.01.76.

34. Feldman BM, Funk SM, Bergstrom B-M, et al. Validation of a new pediatric joint scoring system from the International Hemophilia Prophylaxis Study Group: validity of the Hemophilia Joint Health Score (HJHS). Arthritis Care Res (Hoboken) 20111 63(2): 223-30. doi: 10.1002/acr.20353.

35. Hilliard P, Funk S, Zourikian N, et al. Hemophilia Joint Health Score reliability study. Haemophilia 2006; 12(5): 518-25. doi: 10.1111/j.1365-2516.2006.01312.x.

36. Feldman BM, Funk $S$, Hilliard $P$, et al., on behalf of the International Prophylaxis Study Group. The Haemophilia Joint Health Score (HJHS) International Validation Study. Abstracts of the XXVIIIth International Congress of the World Federation of Hemophilia. Haemophilia 2008; 14(Suppl. 2): 83 \# 12 PO 85. doi: 10.1111/j.1365-2516.2008.01725.x.

37. Hilliard P, B.V., Doria A, Blanchette C, Hang M, Feldman BM. The Hemophilia Joint Health Score (HJHS) correlates highly with radiographic damage. Abstracts of the XXVIIIth International Congress of the World Federation of Hemophilia. Haemophilia 2008; 14(Suppl. 2): 80 \#12 PO 56. doi: 10.1111/j.1365-2516.2008.01725.x.

38. Krishnan S, Vietri J, Furlan R, Duncan N. Adherence to prophylaxis is associated with better outcomes in moderate and severe haemophilia: results of a patient survey. Haemophilia 2015; 21(1): 64-70. doi: 10.1111/hae.12533.

39. Schrijvers LH, Beijlevet van der Zande M, Peters M, et al. Adherence to prophylaxis and bleeding outcome in haemophilia: a multicentre study. Br J Haematol 2016; 174(3): 454-60. doi: 10.1111/bjh.14072.

40. Ho S, Gue D, McIntosh K, Bucevska M, Yang M, Jackson S. An objective method for assessing adherence to prophylaxis in adults with severe haemophilia. Haemophilia 2014; 20(1): 3943. doi: 10.1111/hae.12235.

41. Duncan N, Kronenberger W, Roberson C, Shapiro A. VERITASPro: a new measure of adherence to prophylactic regimens in haemophilia. Haemophilia 2010; 16(2): 247-55. doi: 10.1111/j.1365-2516.2009.02129.x.

42. García-Dasí M, Aznar JA, Jiménez-Yuste V, et al. Adherence to prophylaxis and quality of life in children and adolescents with severe haemophilia A. Haemophilia 2015; 21(4): 458-64. doi: 10.1111/hae.12618.

43. Schrijvers LH. Exploring self-management and adherence in haemophilia. Thesis, Universiteit Utrecht, 2015. Available from https://dspace.library.uu.n//handle/1874/319405 (accessed 15 June 2020).

44. Iorio A, Keepanasseril A, Foster G, et al.; WAPPS-Hemo co-investigator network. Development of a web-accessible population pharmacokinetic service - hemophilia (WAPPSHemo): study protocol. JMIR Res Protoc 2016; 5(4): e239. doi: 10.2196/resprot.6558.

HOW TO CITE THIS ARTICLE:

Strike K, Chan A, Iorio A, Maly MR, Stratford PW, Solomon

P. Predictors of treatment adherence in patients with

chronic disease using the Multidimensional Adherence

Model: unique considerations for patients with

haemophilia. J Haem Pract 2020; 7(1): 92-101. https://doi. org/10.17225/jhp00152.

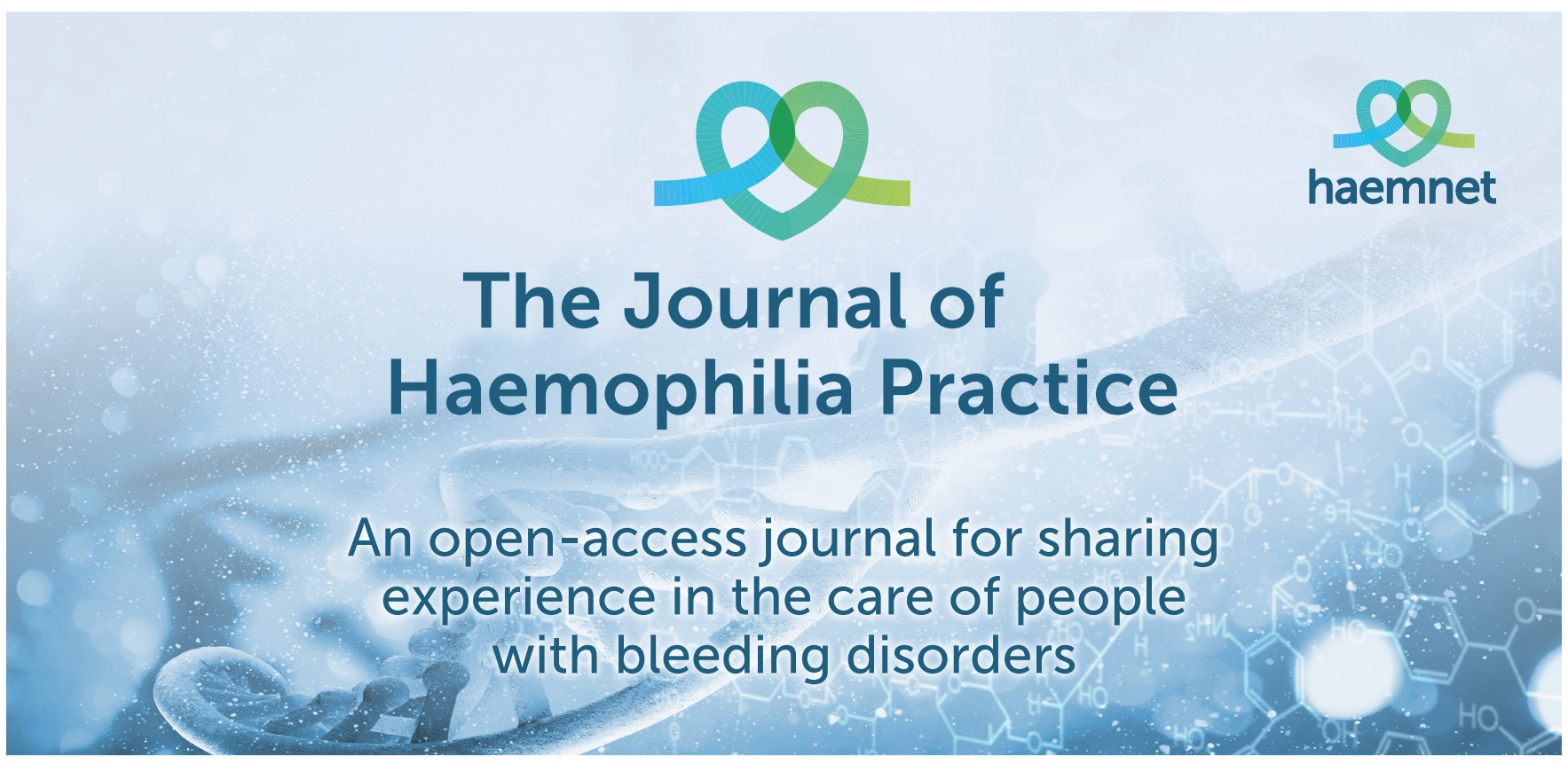

